Supporting Information for Publication

\title{
Modulating the Electron Affinity of Small Bipyridyl Molecules on Single Gold Nanoparticles for Plasmon-Driven Electron Transfer
}

Emily A. Sprague-Klein, ${ }^{\dagger}$ Rosina Ho-Wu, ${ }^{\dagger}$ Duc Nguyen, ${ }^{\dagger}$ Scott C. Coste, ${ }^{\dagger}$ Yue Wu, ${ }^{\dagger}$ John J. McMahon, 5 Tamar Seideman, ${ }^{\dagger \dagger}$ George C. Schatz, ${ }^{\dagger \dagger *}$ Richard P. Van Duyne $\$ \dagger$

†Applied Physics Graduate Program and $†$ Department of Chemistry, Northwestern University, Evanston, IL 60201

USA $\varsigma$ Department of Chemistry, Fordham University, Rose Hill Bronx, NY 10458 USA

${ }^{\beta}$ Deceased July $28^{\text {th }}, 2019{ }^{*}$ Corresponding author. E-mail: g-schatz@northwestern.edu

Table of Contents:

- Figure S1: Solution phase SERS measurements of aggregated $90 \mathrm{~nm}$ gold cores with five pyridine derivative molecules (22BPY, 44BPY, BPA, BPE, and BPEt)

- Figure S2: Correlated LSPR scattering and TEM imaging of single dimer particles.

- Figure S3: Correlated LSPR scattering and TEM imaging of single trimer particles.

- Figure S4: Correlated LSPR scattering and TEM imaging of larger multi-core single particles.

- Figure S5: Additional time-dependent spectral data for the 22BPY radical anion at $532 \mathrm{~nm}$ and $594 \mathrm{~nm}$ pump excitation wavelengths.

- Figure S6: Additional time-dependent spectral data for the 44BPY radical anion at $532 \mathrm{~nm}$ and $594 \mathrm{~nm}$ pump excitation wavelengths.

- Figure S7: Additional time-dependent spectral data for the BPA radical anion at $532 \mathrm{~nm}$ and $594 \mathrm{~nm}$ pump excitation wavelengths.

- Figure S8: Additional time-dependent spectral data for the BPEt radical anion at $532 \mathrm{~nm}$ and $594 \mathrm{~nm}$ pump excitation wavelengths.

- Figure S9: TDDFT calculations for the bare $\mathrm{Au}_{20}$ cluster.

- Figure S10: TDDFT calculations for gas phase BPA.

- Figure S11: TDDFT calculations for surface-bound BPA to $\mathrm{Au}_{20}$.

- Figure S12: TDDFT calculations for gas phase BPE.

- Figure S13: TDDFT calculations for surface-bound BPE to $\mathrm{Au}_{20}$.

- Figure S14: TDDFT calculations for gas phase 44bpy.

- Figure S15: TDDFT calculations for surface-bound 44bpy to $\mathrm{Au}_{20}$.

- Figure S16: TDDFT calculations for gas phase 22bpy.

- Figure S17: TDDFT calculations for surface-bound 22bpy to $\mathrm{Au}_{20}$.

- Figure S18: TDDFT calculations for gas phase BPEt.

- Figure S19: TDDFT calculations for surface-bound BPEt to $\mathrm{Au}_{20}$. 
Figure S1:

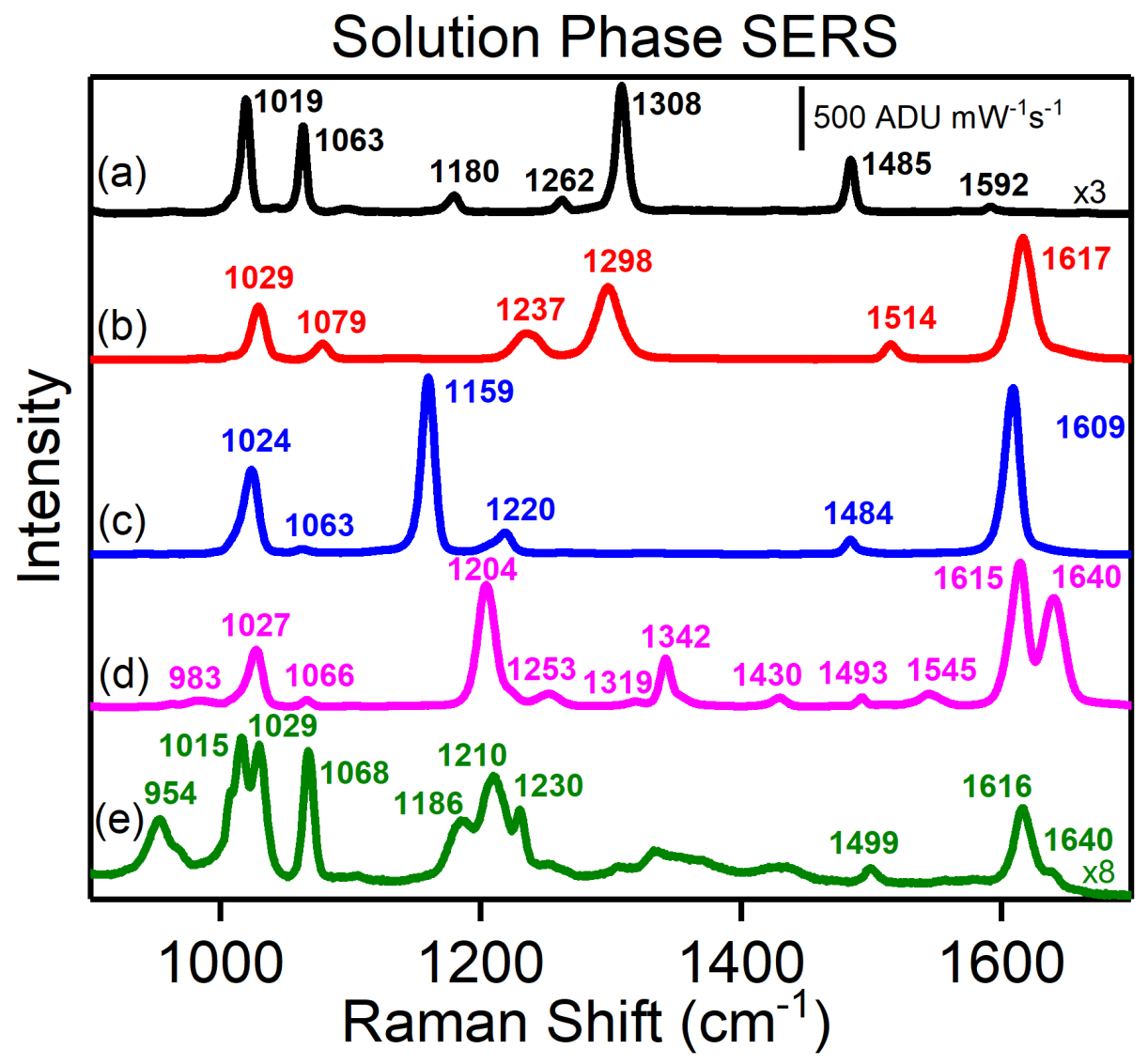

Figure S1. Solution phase SERS functionalized with various pyridine derivative adsorbates a) 22BPY b) 44BPY c) BPA d) BPE and e) BPEt on $90 \mathrm{~nm}$ gold cores. 
Figure S2:
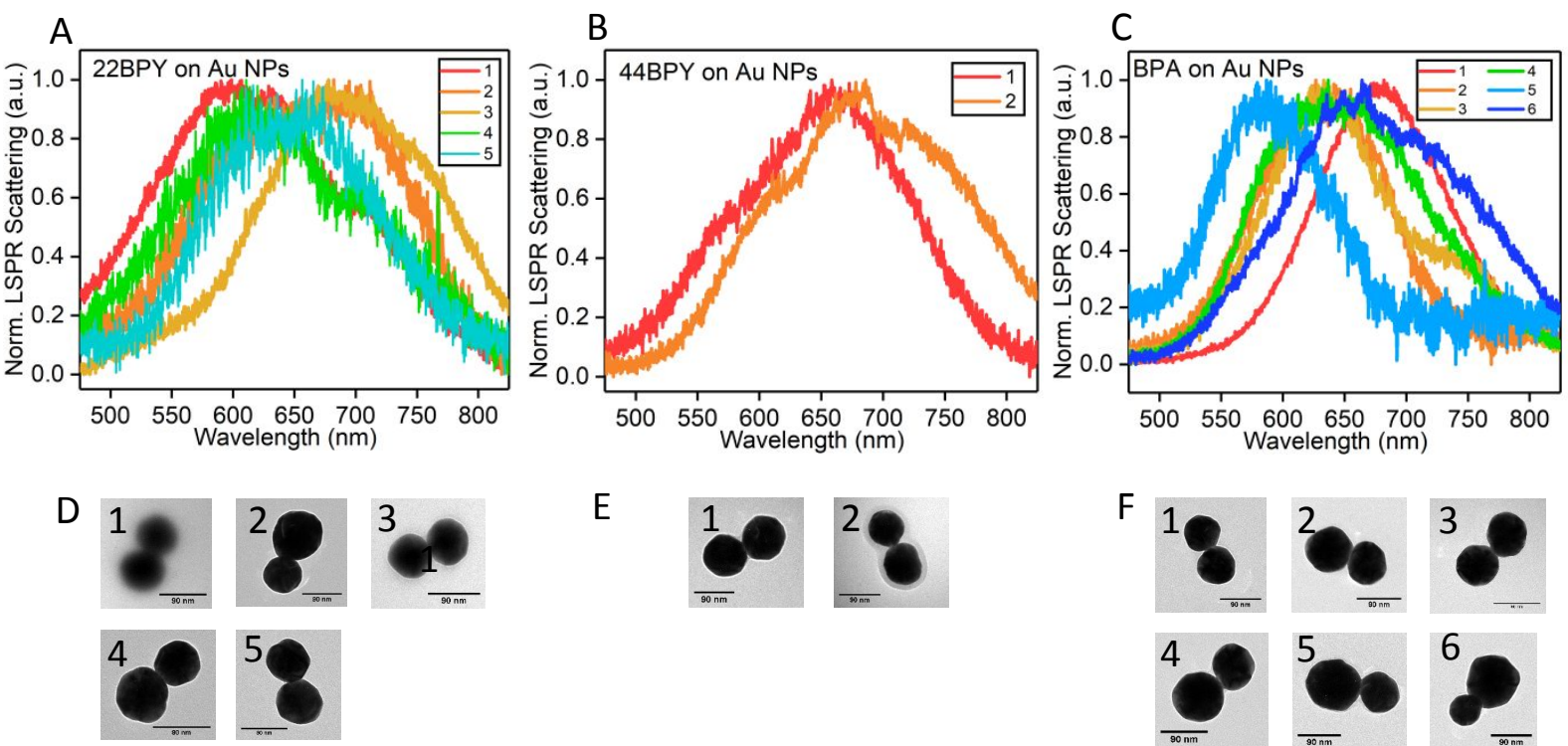

$E$
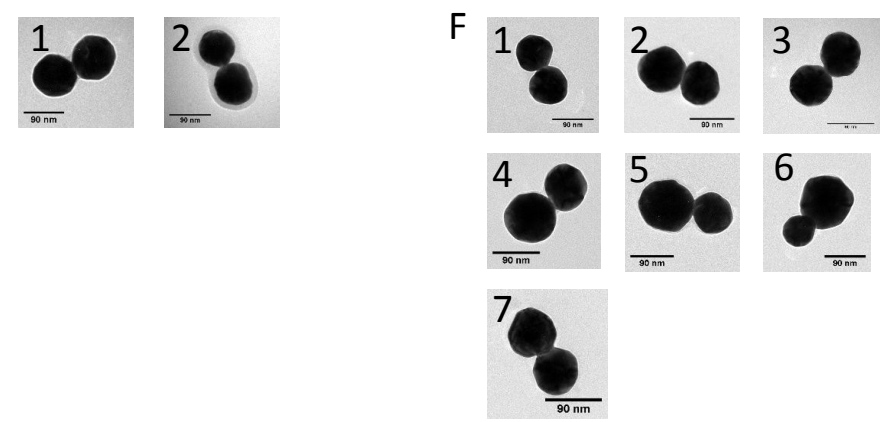

Figure S2. Single particle LSPR scattering profiles for dimers functionalized with various pyridine derivative adsorbates a) 22BPY b) 44BPY and c) BPA on $90 \mathrm{~nm}$ gold cores. Correlated TEM images of dimer particle geometries for d) 22BPY e) 44BPY and f) BPA reporter molecules are shown. 
Figure S3:

A

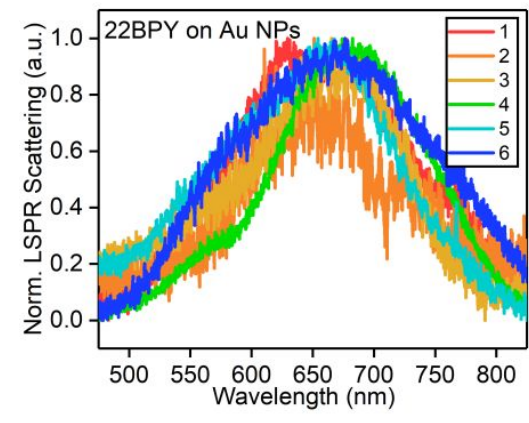

D 1
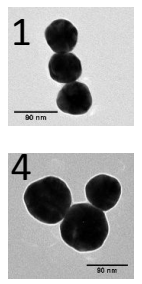

B

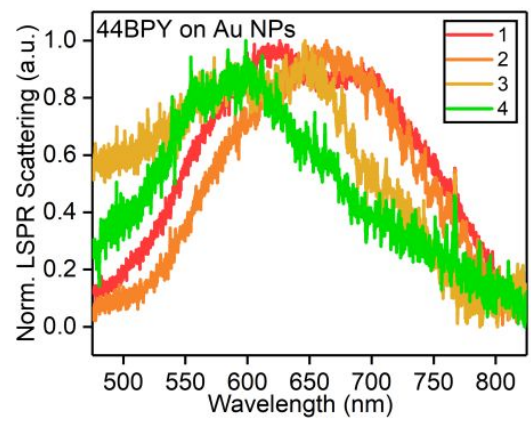

E 1
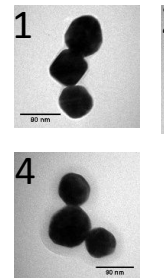

$\mathrm{C}$

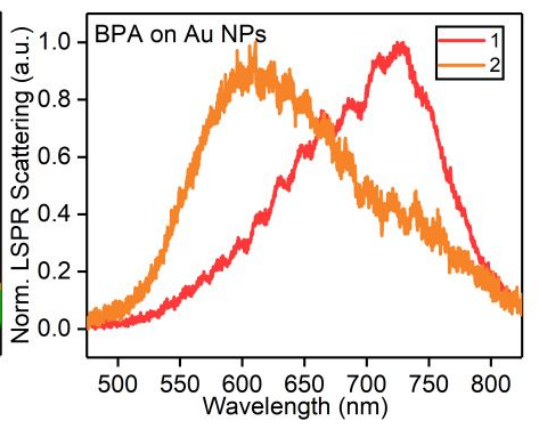

$\mathrm{F}$

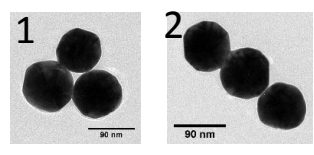

Figure S3. Single particle LSPR scattering profiles for trimers functionalized with various pyridine derivative adsorbates a) 22BPY b) $44 \mathrm{BPY}$ and c) BPA on $90 \mathrm{~nm}$ gold cores. Correlated TEM images of trimer particle geometries for d) 22BPY e) 44BPY and f) BPA reporter molecules are shown. 
Figure S4:
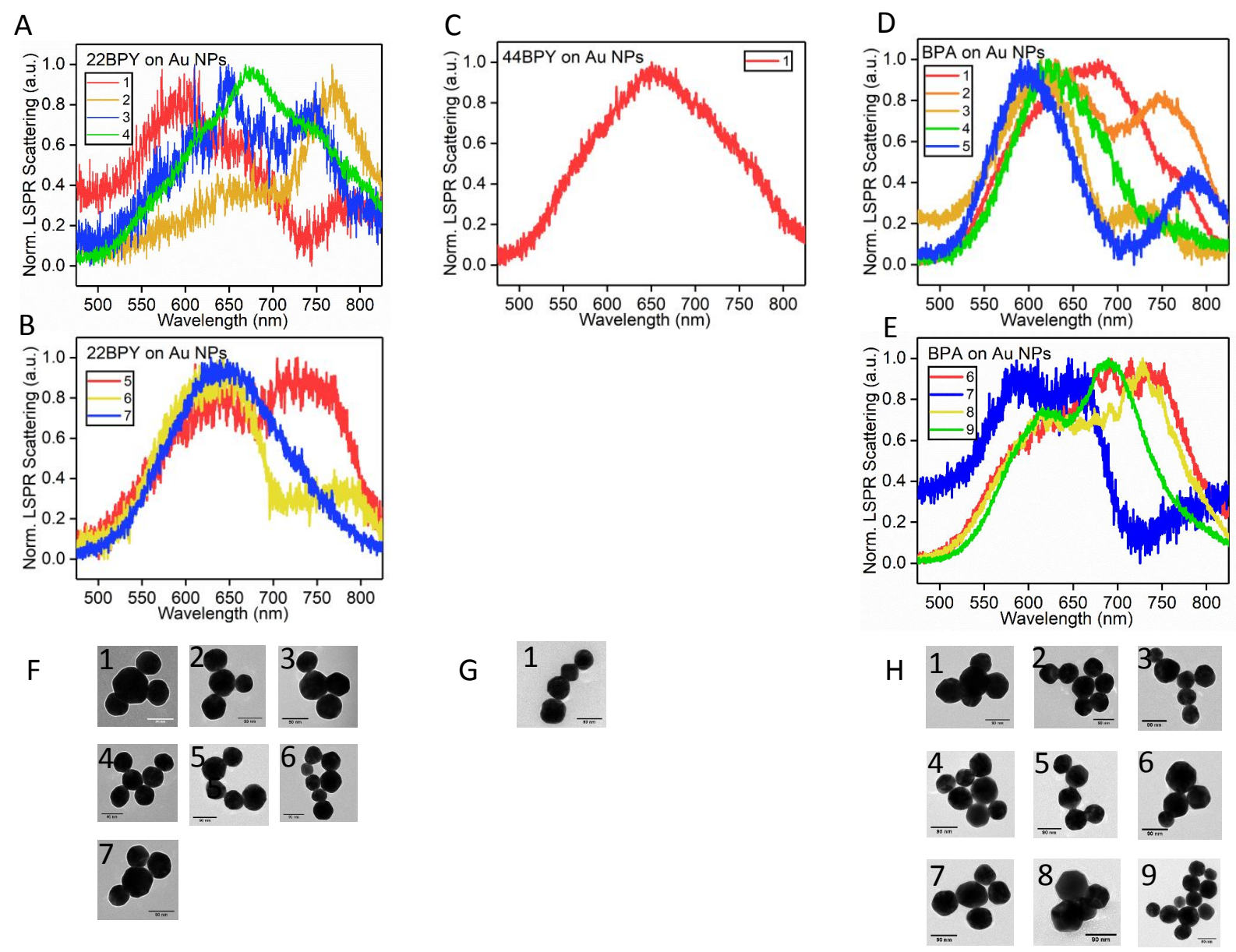

Figure S4. Single particle LSPR scattering profiles for multi-core aggregate particles functionalized with various pyridine derivative adsorbates a)-b) 22BPY c) 44BPY and d)-e) BPA on $90 \mathrm{~nm}$ gold cores. Correlated TEM images of multi-aggregate particle geometries for f) 22BPY g) 44BPY and h) BPA reporter molecules are shown. 
Figure S5:
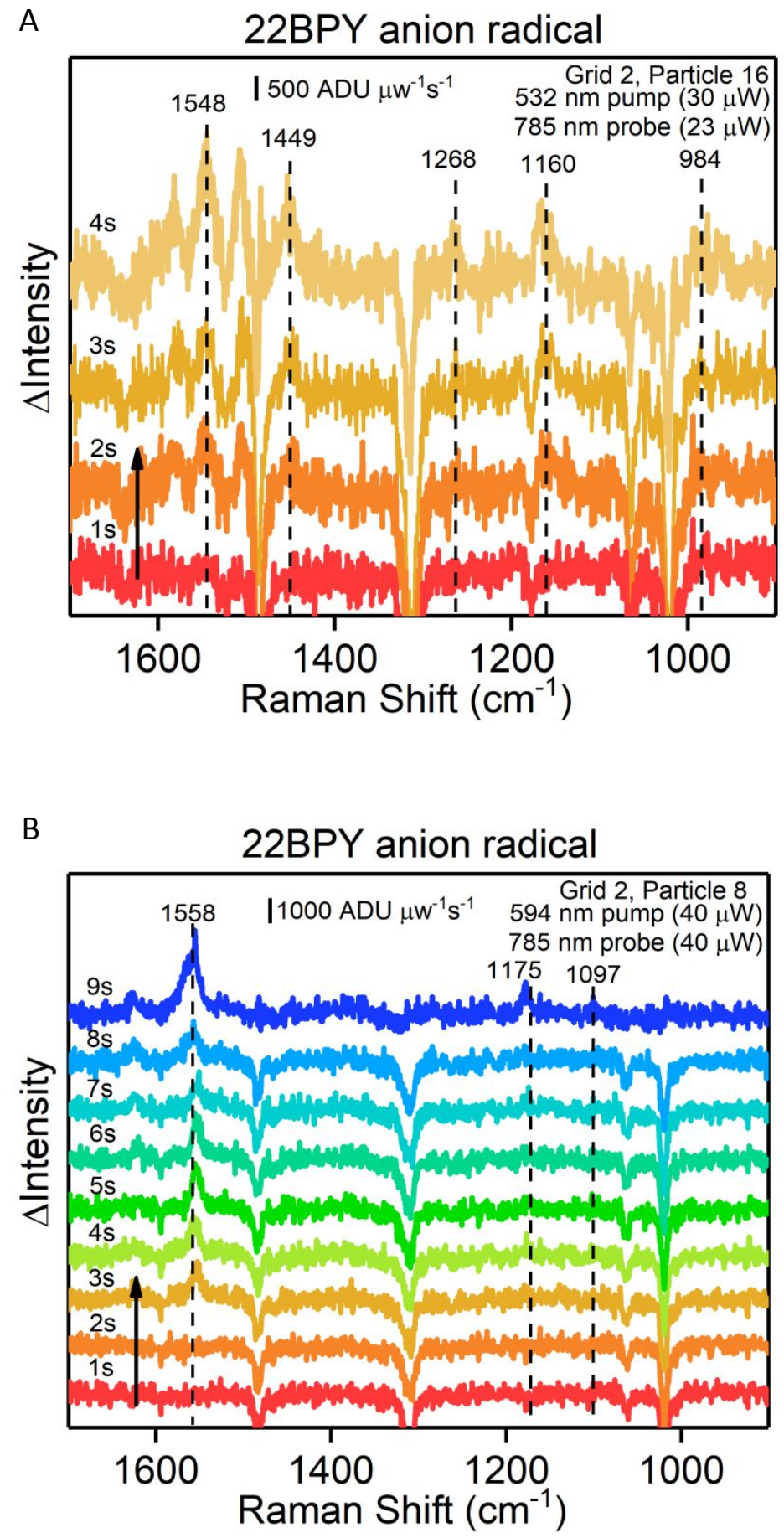

Figure S5. Single particle time-series SERS data for a) $532 \mathrm{~nm}$ pump and b) $594 \mathrm{~nm}$ pump both probed at $785 \mathrm{~nm}$ on 22BPY functionalized $90 \mathrm{~nm}$ gold nanospheres. 
Figure S6:

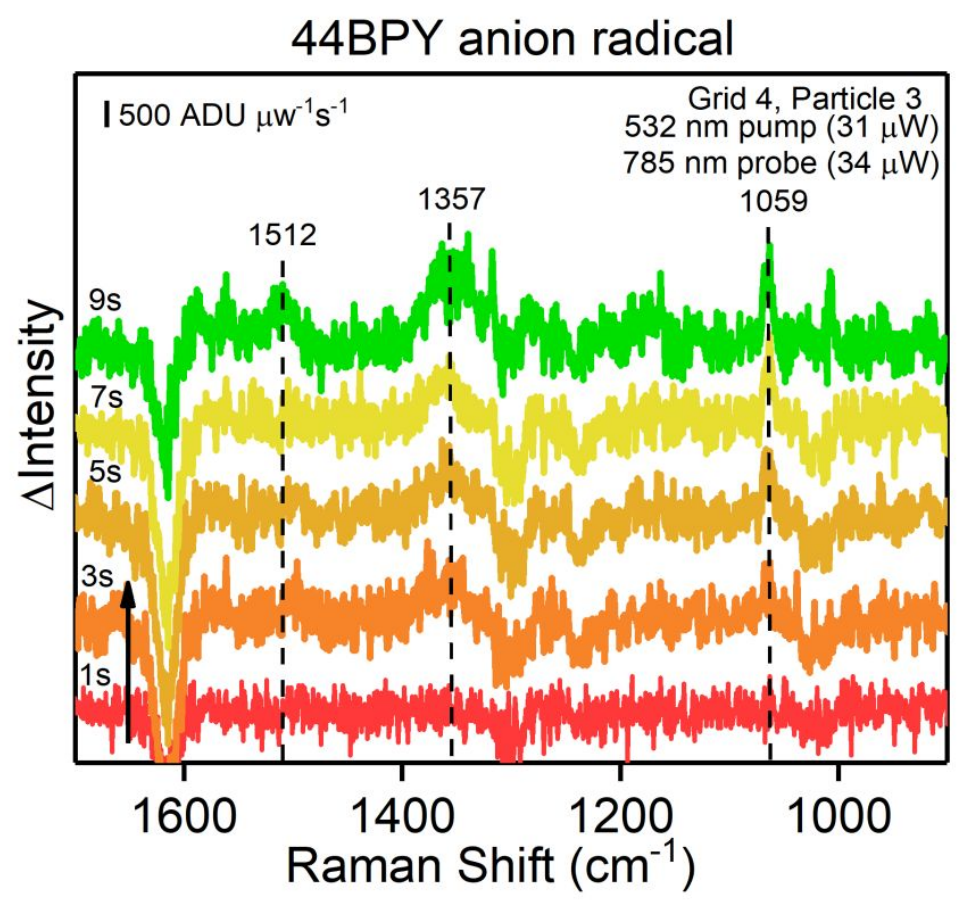

Figure S6. Single particle time-series SERS data for $532 \mathrm{~nm}$ pump and $785 \mathrm{~nm}$ probe on 44BPY functionalized $90 \mathrm{~nm}$ gold nanospheres. 
Figure S7:
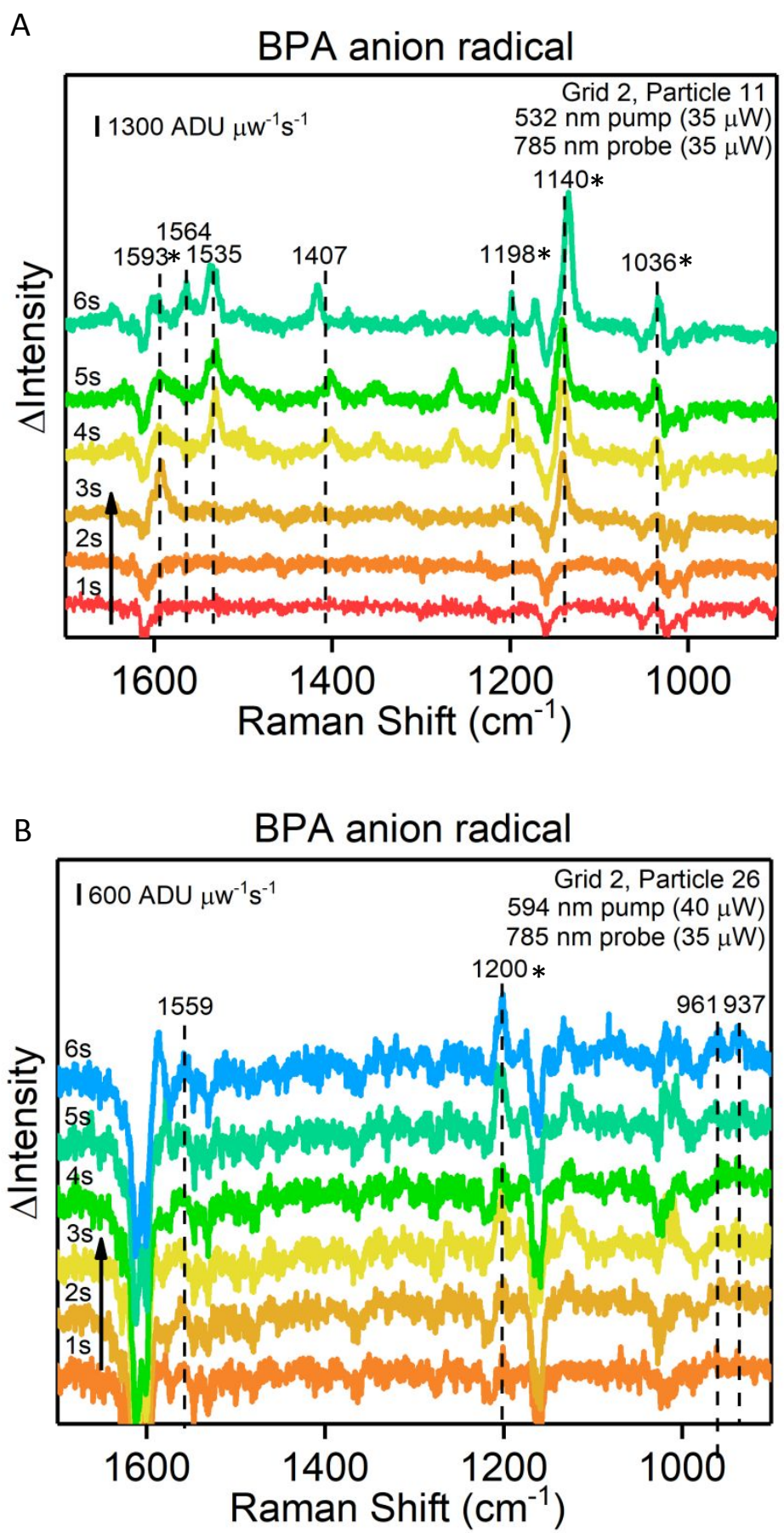

Figure S7. Single particle time-series SERS data for a) $532 \mathrm{~nm}$ pump and b) $594 \mathrm{~nm}$ pump both probed at $785 \mathrm{~nm}$ on BPA functionalized $90 \mathrm{~nm}$ gold nanospheres. Modes designated with an asterisk $(*)$ are assigned as neutral species modes. 
Figure S8:

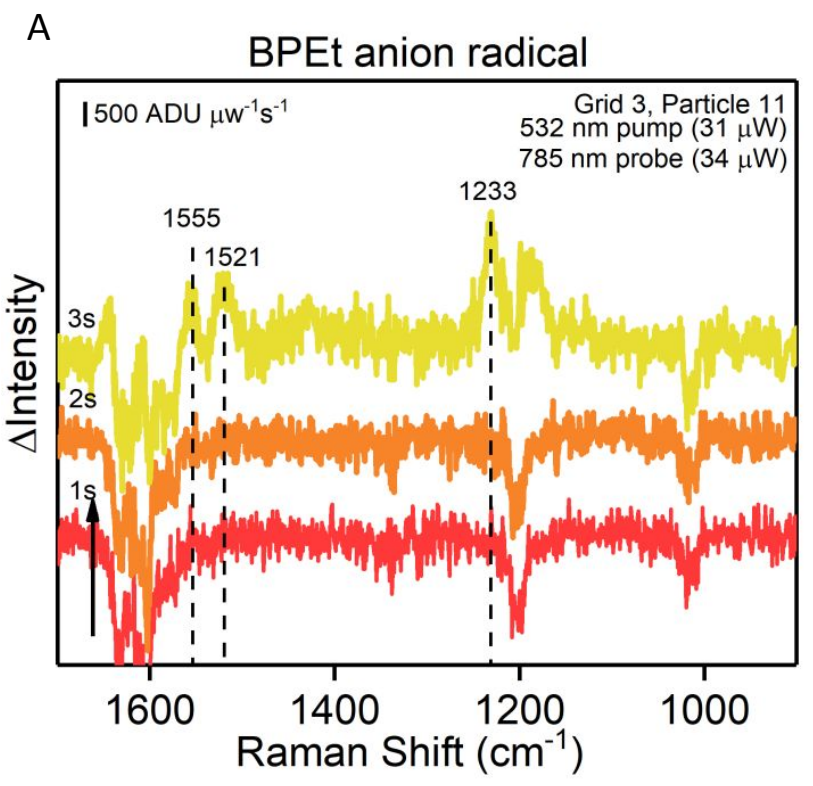

B

BPEt anion radical

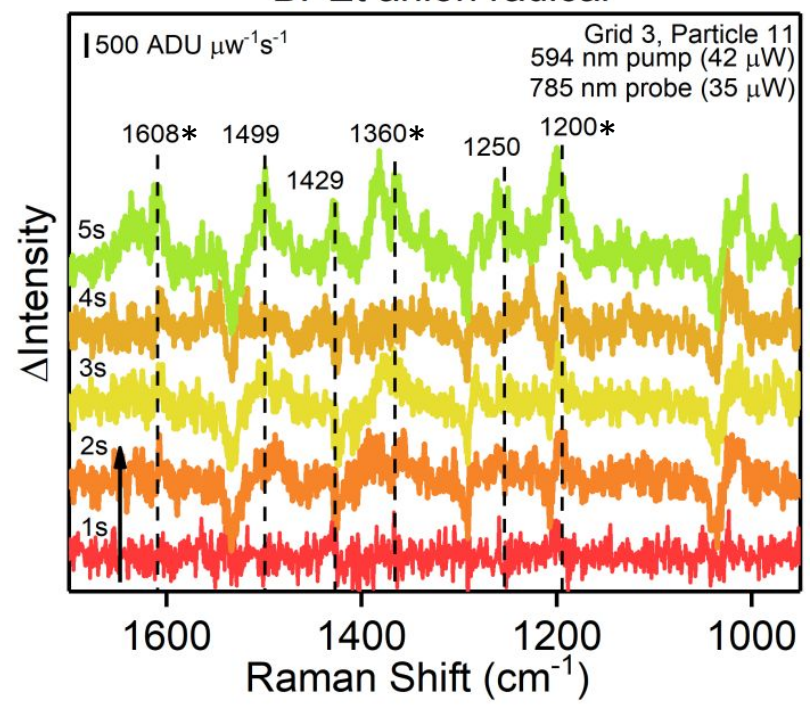

Figure S8. Single particle time-series SERS data for a) $532 \mathrm{~nm}$ pump and b) $594 \mathrm{~nm}$ pump both probed at $785 \mathrm{~nm}$ on BPEt functionalized $90 \mathrm{~nm}$ gold nanospheres. Modes designated with an asterisk $(*)$ are assigned as neutral species modes. 
Figure S9:

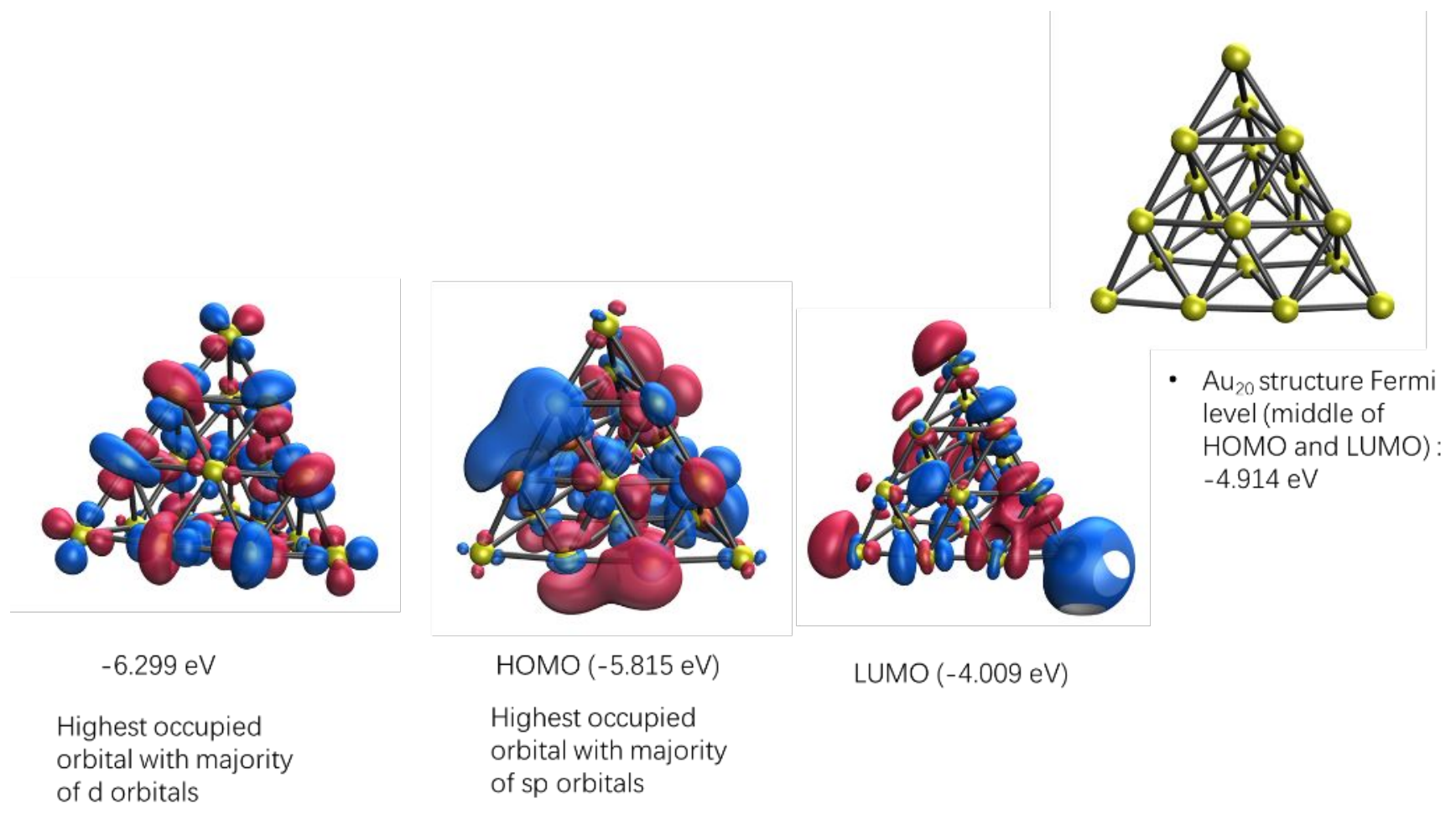

Figure S9. Energetic orbital TDDFT calculations for bare $\mathrm{Au}_{20}$. 
Figure S10:

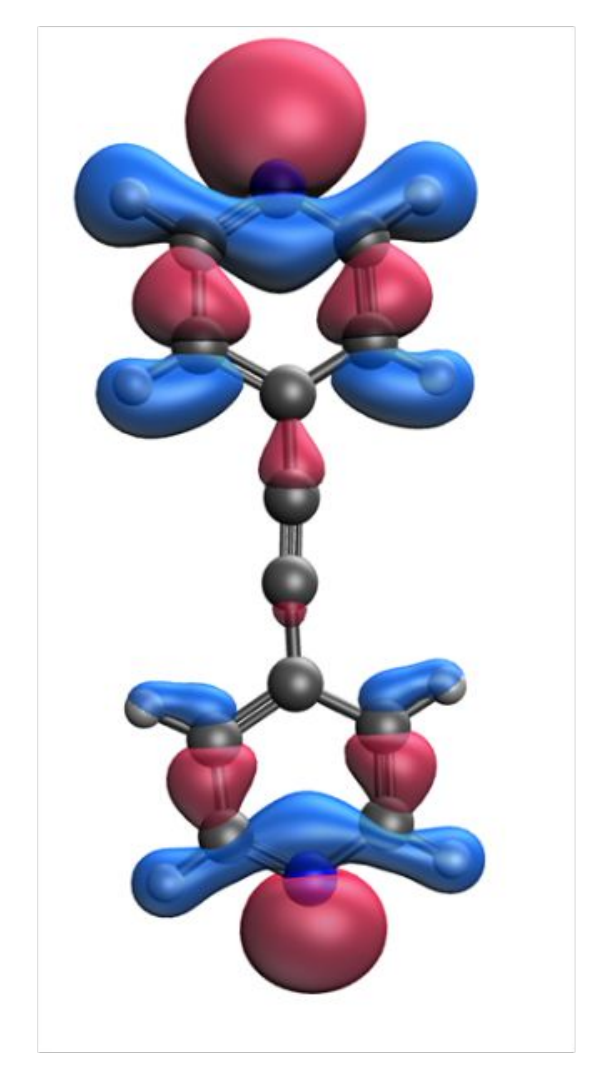

$\mathrm{HOMO}(-6.348 \mathrm{eV})$

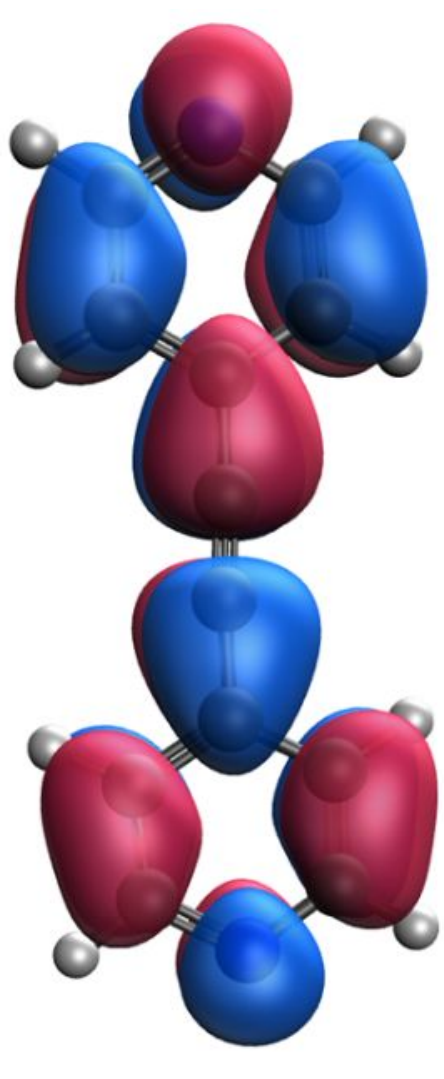

LUMO (-3.618 eV)

Figure S10. Gas phase TDDFT calculations for the BPA molecule. 
Figure S11:

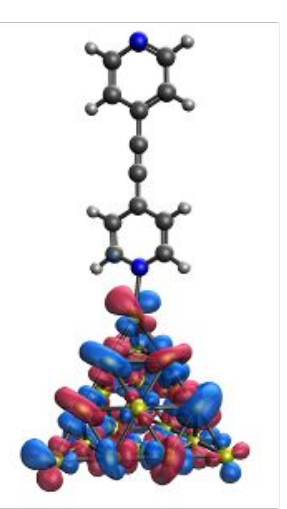

$-5.405 \mathrm{eV}$

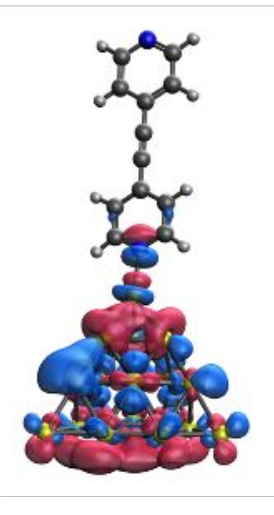

HOMO (-4.984 eV)

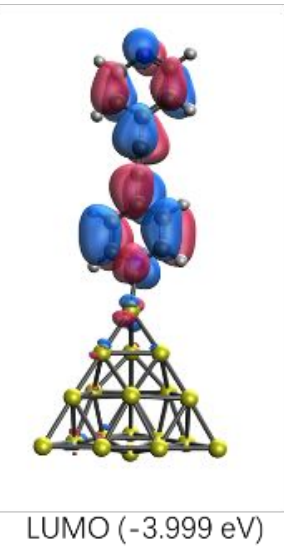

Highest occupied

Au sp orbitals

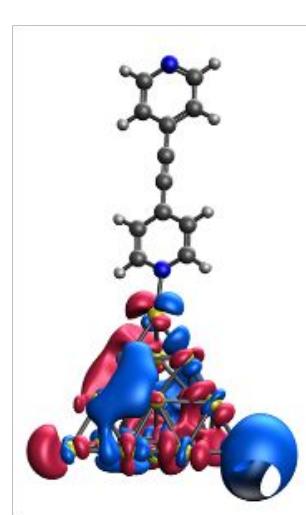

LUMO+1 (-3.541 eV)

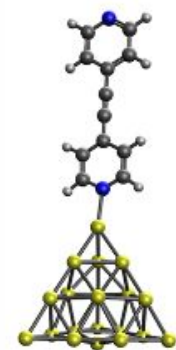

- Fermi level (middle of $\mathrm{HOMO}$ and $\mathrm{LUMO}+1):-4.262$ $\mathrm{eV}$
Au d orbitals

Figure S11. Surface vertex bound BPA-Au ${ }_{20}$ calculations for energy levels using TDDFT. 
Figure S12:

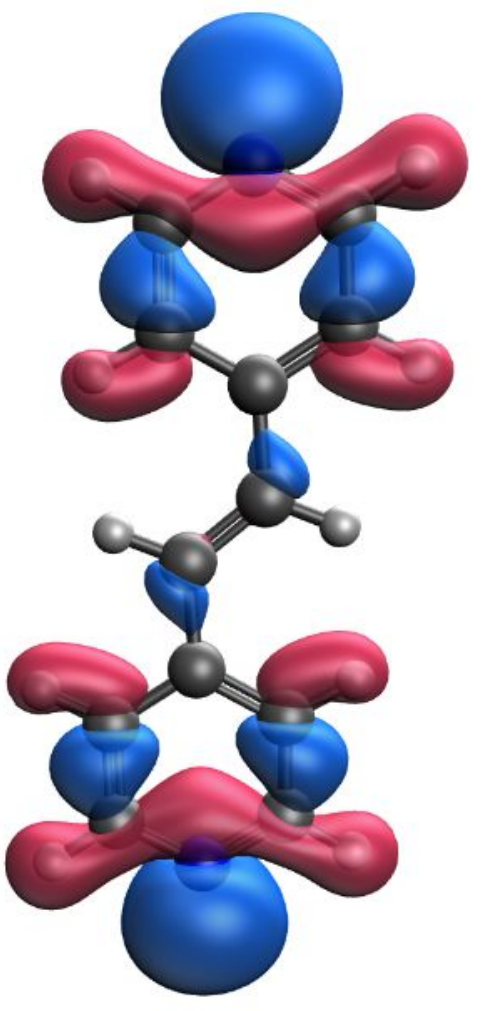

HOMO (-5.920 eV)

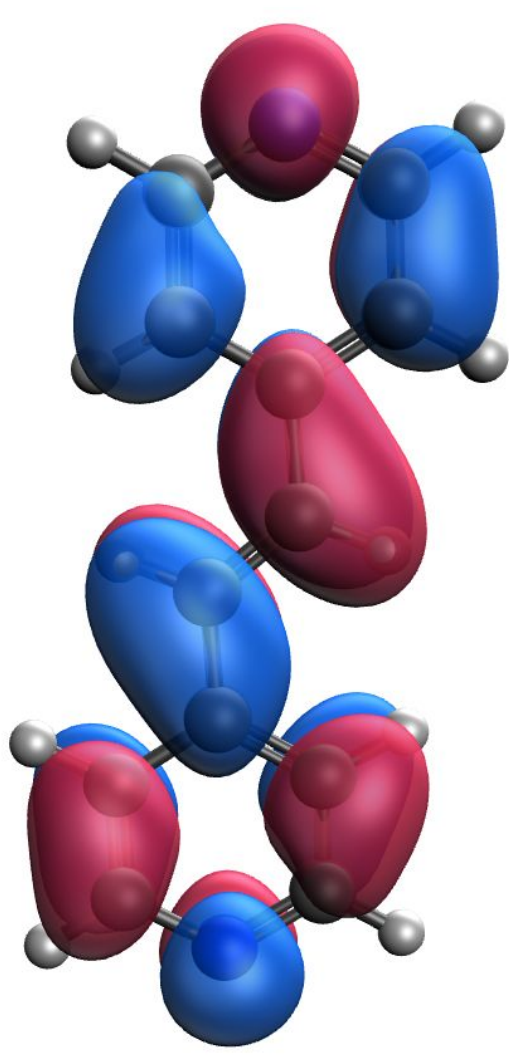

LUMO (-3.207 eV)

Figure S12. Gas phase TDDFT calculations for the BPE molecule. 
Figure S13:

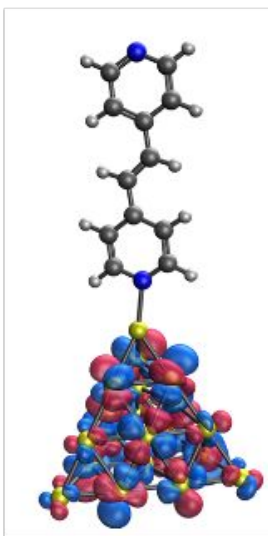

$-5.528 \mathrm{eV}$

Highest occupied Au d orbitals

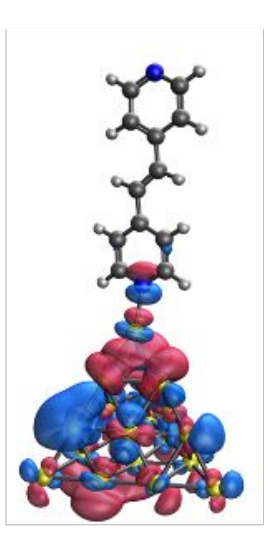

HOMO (-5.009 eV)

Highest occupied Au sp orbitals

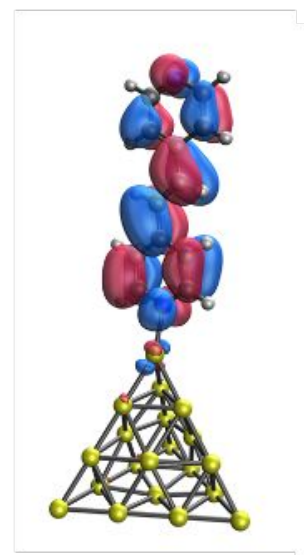

LUMO (-4.095 eV)

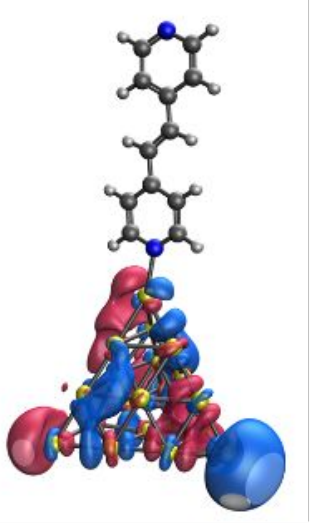

LUMO+1 (-3.573 eV)

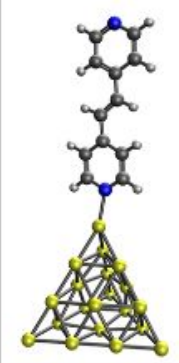

- Fermi level (middle of $\mathrm{HOMO}$ and LUMO+1): -4.291 eV

Figure S13. Surface vertex bound $\mathrm{BPE}-\mathrm{Au}_{20}$ calculations for energy levels using TDDFT. 
Figure S14:
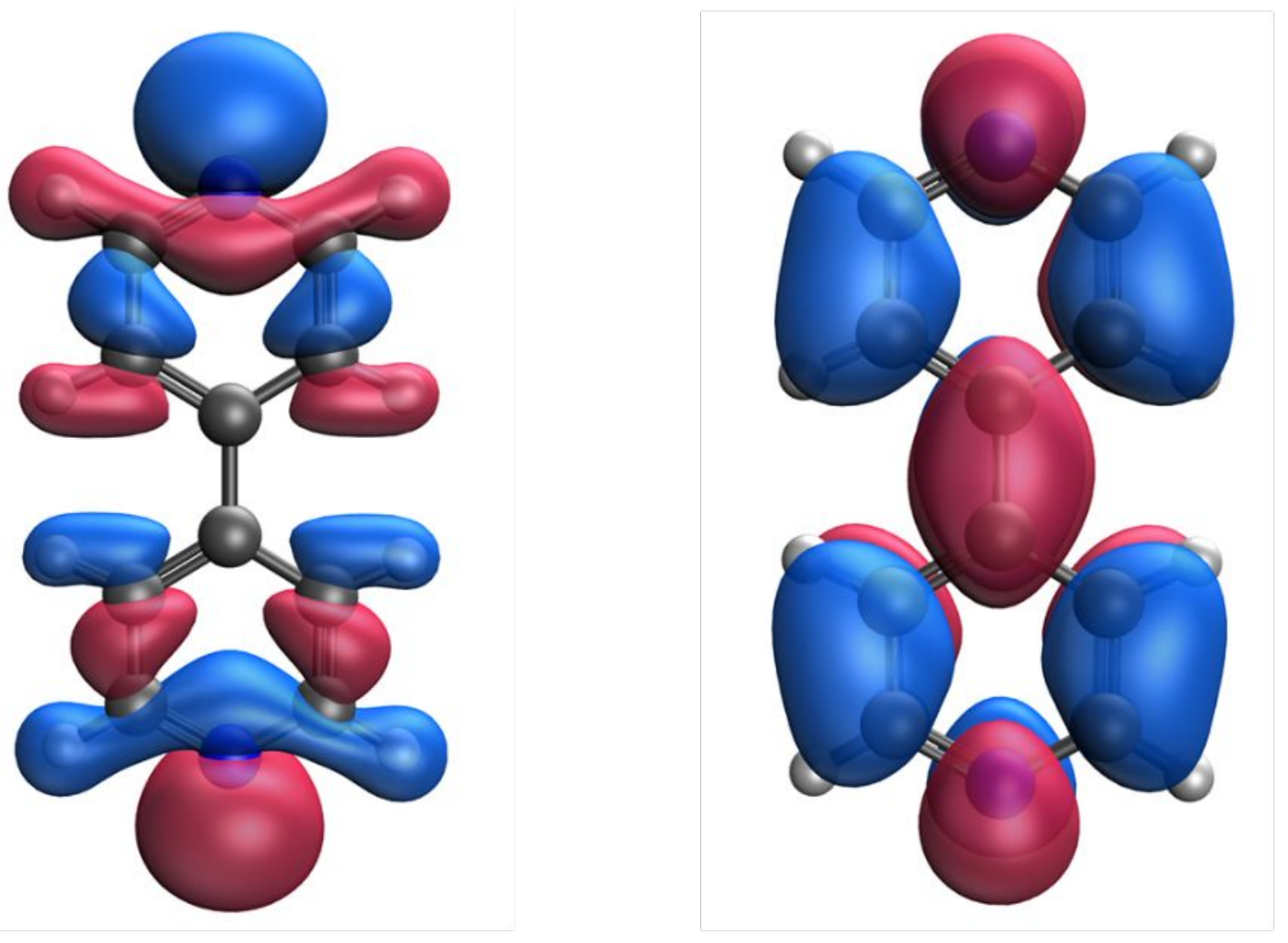

HOMO (-5.962 eV)

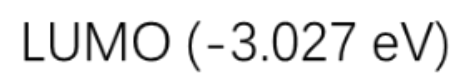

Figure S14. Gas phase TDDFT calculations for the 44bpy molecule. 
Figure S15:

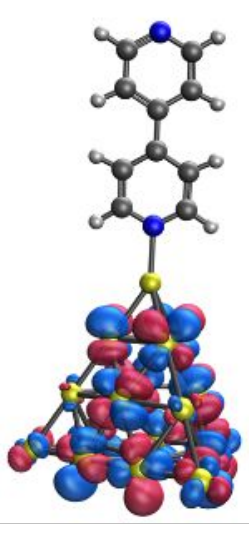

$-5.523 \mathrm{eV}$

Highest occupied Au d orbitals

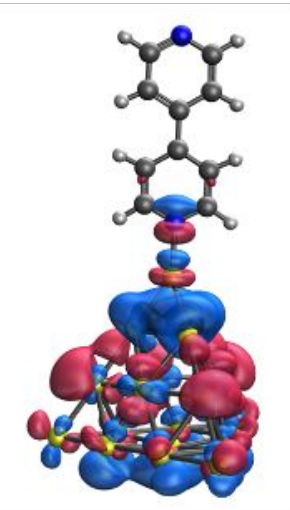

HOMO (-4.994 eV)

Highest occupied

Au sp orbitals

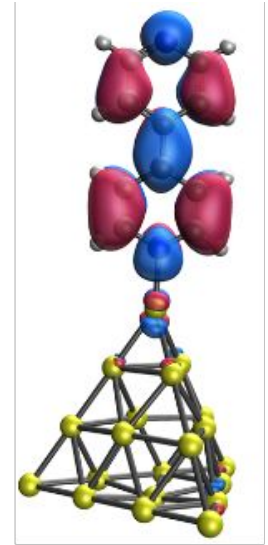

LUMO (-4.048 eV)

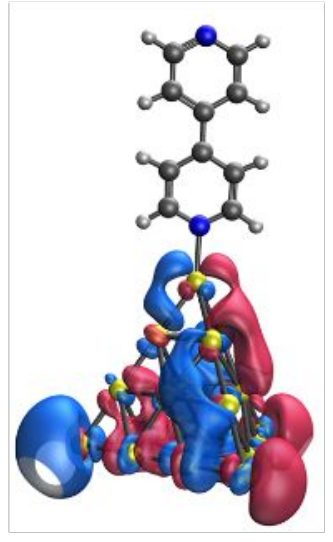

LUMO +1 $(-3.570 \mathrm{eV})$
- Fermi level (middle of $\mathrm{HOMO}$ and LUMO+1): -4.282 $\mathrm{eV}$

Figure S15. Surface vertex bound 44bpy- $\mathrm{Au}_{20}$ calculations for energy levels using TDDFT. 
Figure S16:

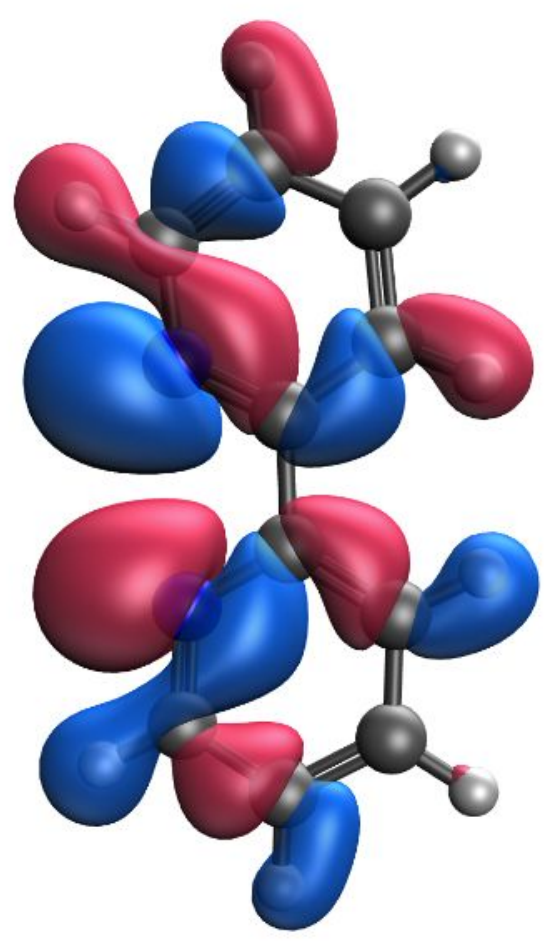

HOMO (-5.415 eV)

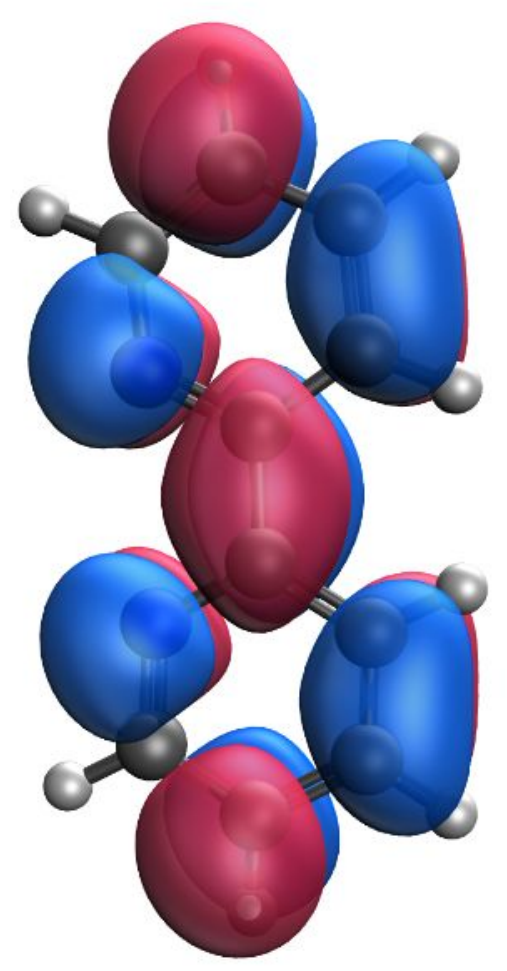

LUMO (-2.607 eV)

Figure S16. Gas phase TDDFT calculations for the 22bpy molecule. 
Figure S17:

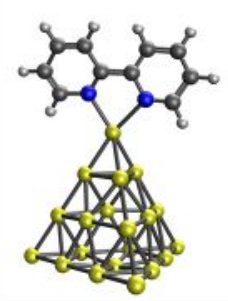

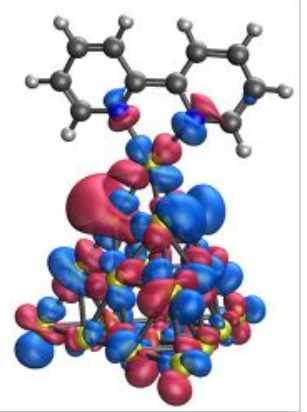

$-5.237 \mathrm{eV}$

Highest occupied Au d orbitals

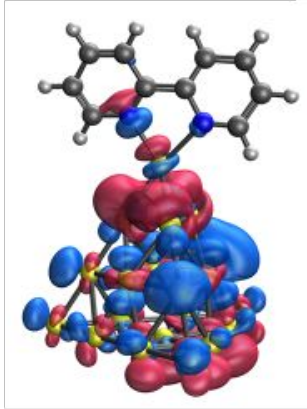

HOMO (-4.806 eV)

Highest occupied

Au sp orbitals
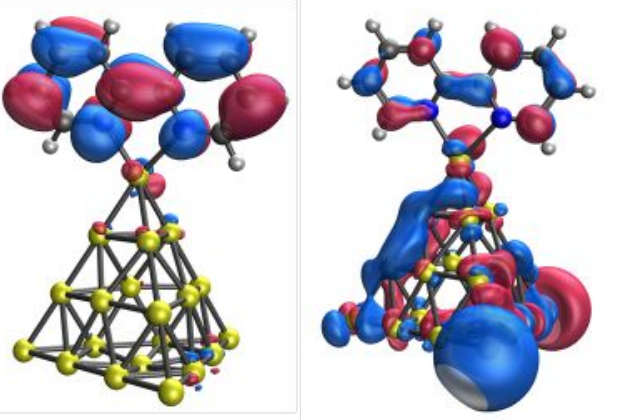

LUMO+1 (-3.343 eV)

Fermi level (middle of $\mathrm{HOMO}$ and LUMO+1) : -4.074 $\mathrm{eV}$

Figure S17. Surface vertex bound 22bpy- $\mathrm{Au}_{20}$ calculations for energy levels using TDDFT. 
Figure S18:

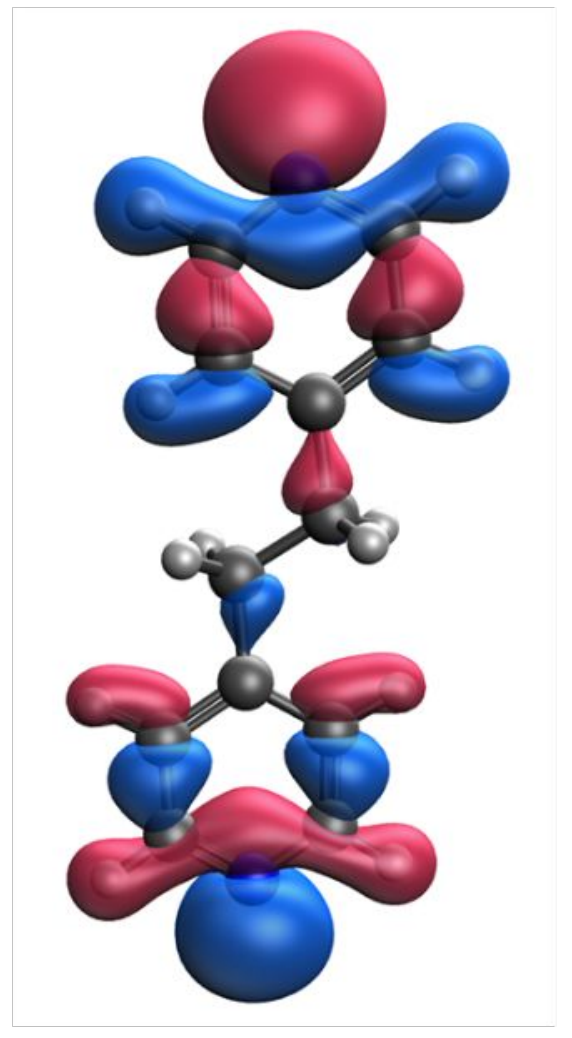

HOMO (-6.714 eV)

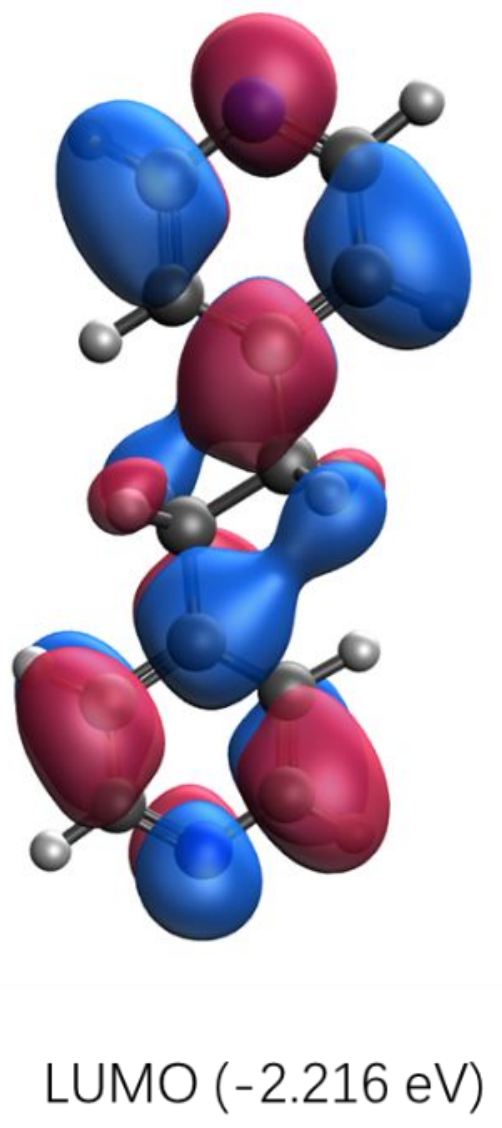

LUMO (-2.216 eV)

Figure S18. Gas phase TDDFT calculations for the BPEt molecule. 
Figure S19:

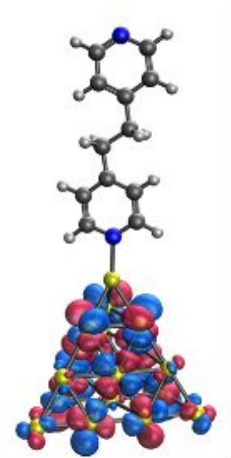

$-5.533 \mathrm{eV}$

Highest occupied Au d orbitals

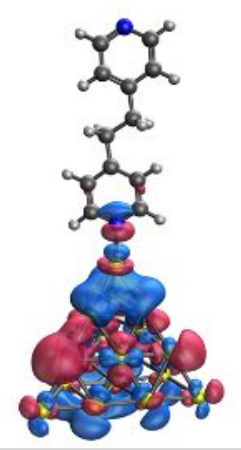

HOMO (-5.005 eV)

Highest occupied Au sp orbitals

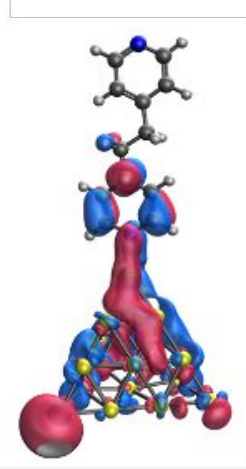

$\operatorname{LUMO}(-3.633 \mathrm{eV})$

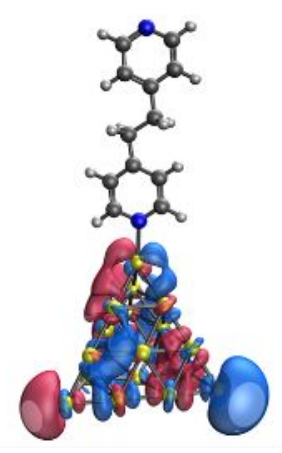

LUMO+1

$(-3.580 \mathrm{eV})$
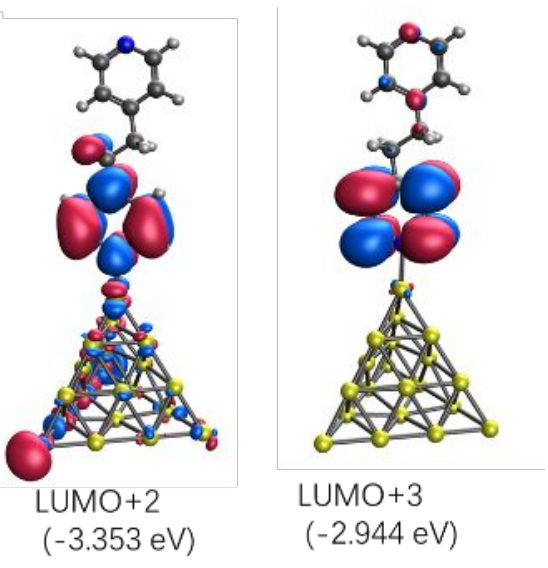

Figure S19. Surface vertex bound BPEt- $\mathrm{Au}_{20}$ calculations for energy levels using TDDFT. 